\title{
Research Status and Development Trend of The Signal Pathway of Allergic Asthma
}

\author{
Hexia LI \\ School of Medicine \\ Anhui University of Science and Technology \\ Huainan, China \\ e-mail: 447162015@qq.com \\ Xinfeng WANG* \\ School of Environment and Resources \\ Xiangtan University \\ Xiangtan, China \\ e-mail: wangxinfeng110@126.com
}

\author{
Chaopin LI \\ School of Medicine \\ Anhui University of Science and Technology \\ Huainan, China \\ e-mail: 1252787767@qq.com \\ Qian LIU \\ School of Medicine \\ Anhui University of Science and Technology \\ Huainan, China \\ e-mail: qiangwang1110@126.com
}

\begin{abstract}
Allergic asthma is a chronic airway inflammatory disease characterized by the infiltration of eosinophils in the lungs and the production of various kinds of stimulating factors, incidence rate showed an upward trend. In this paper, the research status and future development trend of the signal pathway of allergic asthma were introduced. The recombinant allergen vaccine DCP-IhC-ProDerf1of 1 kinds of allergen PrDerf1 in the experiment is put forward, which is used to investigate the effect of specific immunotherapy on the vaccine specific immunotherapy and the related pathway PI3K/AKT in asthmatic mice. Pathway mechanism provides a new idea for specific immunotherapy of allergic asthma caused by dust mites, which has a certain theoretical significance and clinical application value, and may be a new promising approach for the treatment of allergic asthma.
\end{abstract}

Keywords-allergic asthma; signal pathway; research status; development trend

\section{INTRODUCTION}

Allergic disease is one of the worldwide public health problems, attracted the attention of health personnel, and is very common in clinical practice and multiple common manifestations of allergic asthma, allergic rhinitis, allergic dermatitis, atopic eczema, allergic purpura. Which allergic asthma is more common and the incidence rate is increasing year by year, causing people's attention, has become a hot spot of research. Allergic asthma is a chronic airway inflammatory disease caused by allergens that are exposed to the air [1].Dust mite is a common cause of allergic asthma, dust mite allergen I is one of the main reasons [2].

Allergic asthma is the most common type of I hypersensitivity reaction characterized by chronic eosinophilic infiltration, airway inflammation, reversible airway obstruction, mucus secretion, and nonspecific airway.There is a lot of evidence show that these inflammatory responses are regulated by Th2 and Th1, together with mast cells, bronchial epithelial cells and eosinophils, and many inflammatory cytokines and chemokines.

\section{RESEARCH SigNIFICANCE}

The present study has showed that in stimulating specific antigen, the immune response of $\mathrm{T}$ cell subsets in Th1/Th2 imbalance and dysfunction is a key mechanism in the pathogenesis of asthma, as Th1 type cytokines (such as IL-12, IFN- $\gamma$ ) reduces the secretion of cytokines before allergic Th2 cells (such as IL-4, IL-5, IL-13) increased and hypersplenism. In addition, Th17/Treg imbalance was also found in asthma patients, that is th17/Treg is also involved in the regulation of allergic asthma. Human studies suggest that allergen specific immunotherapy (Allegan Special Immunotherapy) is the only cause of allergic diseases prevention and effective treatment method at present, can effectively balance the Th1/Th2 imbalance, thereby inhibiting the secretion of Th2 cytokines, promoting the secretion of Th1 cytokines, and can effectively inhibit the secretion of IgE. Reduce the generation of inflammatory cells [3-4]. But because the vaccine is used for ASIT crude extract, there are often unknown allergen characteristics, the vaccine composition is complex, and the amount of vaccine is also high, easy to cause some adverse reactions. Therefore, the new vaccine for ASIT is the development of vaccines with low allergen and high immunogenicity. In addition, the pathogenesis of asthma is more complex, and it is affected by the double factors of heredity and environment, especially the polymorphism of MHCII molecule, which limits the wide application of ASIT.

In recent years, through the MHCII way to design vaccine has received much attention, compared with the natural allergen, the vaccine can significantly enhance the efficiency of antigen presentation by MHCII way, and it is the effective strategy for allergic asthma ASIT. The current research on allergic asthma pathway has become a hot spot, Hye-Rin Kim, Dong-Min Lee to observe the chlorogenic acid (CGA) on ovalbumin (OVA) inhibitory effects induced by allergic asthma mice found that activation of a variety of molecules including STAT-6, NF- and JNK K B, ERK1/2. These molecules, subject to IL-4/LPS activation of STAT-6 
and JNK were inhibited by CGA [5]. In addition, the upregulation of Derf2 stimulated IL-13 expression through the activation of PI3K/Akt pathway, and further activate the transcription of NF-B pathway and induce the expression of IL-13.The recombinant allergen vaccine DCP-IhCProDerf1of 1 kinds of allergen PrDerf1 in the experiment is put forward, which is used to investigate the effect of specific immunotherapy on vaccine specific immunotherapy and the related pathway PI3K/AKT in asthmatic mice. Pathway mechanism provides a new idea for specific immunotherapy of allergic asthma caused by dust mites, which has a certain theoretical significance and clinical application value.

\section{RESEARCH STATUS AT HOME AND ABROAD}

Allergic disease was WHO as one of the three major diseases in twenty-first Century, one of the major diseases in the world, the total incidence of up to $10 \% \sim 30 \%$. The allergic asthma is common and multiple, is affected by genetic and environmental factors mediated double occurred in each stage of human age a pulmonary eosinophilic infiltration and the excitation factor induce airway hyperresponsiveness characterized by chronic airway inflammatory disease, is a disease of type I allergy mediated by IgE the guide, the incidence is rising year after year. Airborne allergens is an important factor to induce allergic asthma, dust mite exists in the human living environment in the dust is the most common airborne allergens, become the focus of human studies [6]. In dozens of dust mite known, most closely with human allergic diseases is mainly dust mite (Dermatophagoides farinae, Derf) and DerP is one of the major allergen of asthma, the main Derf1/ Derf2 and Derp1/Derp2, and can be as high as $70 \%$ with dust mite allergic patients serum IgE rate.Th1 cells produce IL-2, IL12 and IFN- $\gamma$, with TDTH cells and TC cell proliferation, differentiation, regulation of cell immune response, promote macrophage phagocytosis of pathogenic microorganisms involved in delayed hypersensitivity. Th2 cells produce IL-4, IL-5, IL-6, IL-10 and IL-13, the role of cytokines in B lymphocytes, mast cells and basophils, lead to specific inflammatory process of asthma occurrence, mediated by the immediate allergic reaction.

Asthma is mainly due to the Th0 bias in the Th2 differentiation leads to the imbalance of Th1/Th2, normal human Th1/Th2 ratio in equilibrium after allergen enters the body to break this balance, promote the activation of Th2, and the secretion of inflammatory cytokines, promote the proliferation of B cells and IgE, recruiting inflammatory cells to the body in the gathering, sensitization, when allergen again into the body, and the IgE binding and activation of basophils and mast cells, which release a variety of bioactive substances[7-8], which cause allergic effect, trigger type I hypersensitivity reaction, allergic inflammation manifests as allergic reaction speed characteristics and allergic inflammation of target organ.

In recent years, with the deepening of research, in the past, the main mechanism of Th1/Th2 imbalance in the pathogenesis of asthma has been considered, in many experimental results have been unable to fully explain the pathogenesis of asthma[9-10].Recent studies have found that Th17/Treg cell balance is also involved in the process of asthma. Th17 cell secretion of IL-17 (including IL-17A and IL-17F) and IL-22, promotes the occurrence of autoimmune diseases and inflammatory diseases, and plays a pivotal role. IL-17 is a pro-inflammatory cytokine that promotes the secretion of IL-8, and then the aggregation of neutrophils. In addition, IL-17 plays an important role in airway remodeling process [11]. Treg cells inhibit inflammation, but also play an important role in maintaining immune balance, inhibit the secretion of inflammatory cytokines, suppression of effectors cells (mast cells, basophils and eosinophils) inflammation, inhibit the production of IgE, thus inhibiting the progress of allergic reaction.

ASIT is the only cause of current therapy can change the allergic disease process, which determine the allergens in allergic patients, through a few times with allergens (sensitization extracted directly from sensitized biological materials in the original) method to produce tolerance, in order to achieve desensitization, its clinical efficacy confirmed in allergic rhinitis and ASIT can not only reduce asthma, allergic sensitization, also can reduce the incidence of asthma after treatment [12]:

(1) ASIT can induce the body to produce allergen specific IgG4, IgG1, at the same time effectively reduce the allergen specific IgE antibody levels, thereby blocking the occurrence of type I hypersensitivity reaction;

(2) Which can reduce the secretion of CD4+ T cells, IL4 induced Th0 cells to Th1 cell differentiation, inhibition to Th2 differentiation?

(3) Which can effectively improve the level of CD4+ CD25+ Tr cells and IL-10 levels in the serum of patients with allergic asthma, and exert the function of negative regulation of immune function?

ASIT therapeutics is the traditional crude allergen extracts, although its therapeutic effect has been affirmed, but because its composition is complicated, it is difficult to standardize, easy to produce side effects in the process of using, including local and systemic adverse effects and produce new allergies, which limits the widespread use of crude allergen. Therefore, the new vaccine design method that can improve the safety and effectiveness of ASIT has emerged, and has aroused people's extensive discussion. The process of $\mathrm{CD} 4+\mathrm{T}$ cell recognition of exogenous antigen, MHC II molecular limit, usually exogenous antigen into the body, first by antigen-presenting cells (APCs) recognition and uptake, and then processed into short peptide fragments containing 10 30 amino acid residues, making it suitable for combination with MHC II molecules, formation the II class -MHC antigen peptide complexes, APCs complexes presenting to CD4+T cells, and the activation of CD4+T cells and their differentiation into different subpopulations of cells secrete various cytokines. Th2 activation and secretion of IL-4 can induce B cell proliferation, differentiation, and then produce IgE, resulting in the occurrence of allergic reactions [13].In addition, the complex formation is a complex process, including antigen degradation, Ii degradation and II MHC transport, etc. The 
degradation of Ii chain, the maturation and transport of II class MHC molecules have an important influence on the number of antigenic peptides II -MHC molecular complexes.

In recent years, many studies have connected with the Ii chain by using different methods, which can promote the antigen presentation of the MHC II pathway. The use of MHC II pathway to design vaccine can effectively enhance the antigen presentation, is an effective strategy for allergic asthma ASIT. DC cells as a potent APC known as the most powerful, in antigen uptake, processing and processing and plays an important role in the process, can effectively promote the specific proliferation of $\mathrm{T}$ cells in specific immune and immune tolerance in the process of antigen is extremely important[14]. Marker researchers can combine with DC specific cell surface molecules in vivo injection of the antigen, directly to the location and antigen presentation to DC cells, which have been confirmed experimentally, could significantly enhance vaccine efficacy, and showed great potential application. This method has been applied to tumor immunotherapy, and has achieved some success.

Therefore, early experiments to contain the peptide sequence of ProDerf1 as the research object, and is named for a specific binding to DC cell surface short peptide peptides DCP 3 (FYPSYHSTPQRP) and the 1 to 110 AA encoding MHC II pathway of MHC molecular chain Ii nucleotide sequence (IhC1-110) fusion construction of recombinant DCP-IhC-ProDer vaccine of F1, and SIT in asthmatic mice, the results showed that the fusion protein vaccine with specific immune therapy has a good effect, but its mechanism is still unclear, therefore, this topic on the disease is through what means to play the role of specific immunotherapy for further discussion.

\section{DeVelopment TREnd And PROSPeCt}

The current research on allergic asthma pathway has become a hot spot, Hye-Rin Kim, Dong-Min Lee to observe the chlorogenic acid (CGA) on ovalbumin (OVA) inhibitory effects induced by allergic asthma mice found that activation of a variety of molecules including STAT-6, NF- and JNK K B, ERK1/2. These molecules, subject to IL-4/LPS activation of STAT- 6 and JNK were inhibited by CGA.In addition; the upregulation of Derf2 stimulated IL-13 expression through the activation of PI3K/Akt pathway, and further activate the transcription of NF- B pathway and induce the expression of IL-13. PI3K, a member of the lipid and threonine/serine kinase family, is a potential target for the development of therapeutic drugs for asthma. PI3K plays a very important role in the pathogenesis of asthma. By regulating the downstream signaling pathway, the cell growth,proliferation and differentiation[15]. AKT (i.e. protein kinase B), a threonine/serine kinase, is a direct downstream effector of PI3K. NF-kappa B is a key nuclear transcription factor that regulates the differentiation and cytokine production of Th2 cells.PI3K and AKT play an important role in the activation of NF- K B in asthma model in many studies, regulation of PI3K / Akt pathway of NF- kappa B has been confirmed. Akt activation of IkBa kinase (IKK), IKK, in turn, makes I nuclear factor kappa Ba phosphorylation, resulting in the presence of I nuclear factor kappa Ba in the cytoplasm and the degradation of I and nuclear factor kappa Ba binding together with the release of NF-kB [16]. IkBa is a kind of inhibitory protein that maintains NF- nuclear factor kappa B in the inactive state. The free state of the NF- transcription factor kappa B from the cytoplasm to the nucleus causes proinflammatory cytokine transcription of Th2 gene. In addition, the PI3K/Akt pathway also plays a crucial role in the activation of eosinophils and $\mathrm{T}$ cells, $\mathrm{B}$ cells and mast cells and immune response.

In recent years, the application of PI3K/Akt/NF-B pathway in disease has attracted widespread attention. Studies show that the oxygen tetracycline through the inhibition of NF kappa B pathway to reduce OVA induced allergic airway inflammation in asthmatic mice. Anti malaria drug artesunate through inhibition of PI3K/Akt pathway to alleviate OVA induced allergic asthma symptoms reported in mice. And research shows that common hormone can reduce endotoxin inflammation by PI3K/Akt/NF- B pathway inhibits ROS mediated activity.

So the recombinant allergen vaccine DCP-IhCProDerf1of 1 kinds of allergen PrDerf1 in the experiment are put forward, which is used to investigate the effect of specific immunotherapy on the vaccine specific immunotherapy and the related pathway PI3K/AKT in asthmatic mice. Pathway mechanism provides a new idea for specific immunotherapy of allergic asthma caused by dust mites, which has a certain theoretical significance and clinical application value, and may be a new promising approach for the treatment of allergic asthma.

\section{ACKNOWLEDGMENT}

This work is supported by Hunan Key Laboratory of Geomechanics and Engineering Safety (Grant No.16GES10) and Xiangtan University Natural Science Foundation (Grant No. 15XZX41).

\section{REFERENCES}

[1] Grundstrom J, Saarne T, Kemi C, et al. Development of a Mouse Model for Chronic Cat Allergen-Induced Asthma. Int Arch Allergy Immunol. 2014,165(3): 195-205.

[2] Liu Z, Jiang Y, Li C. Design of a ProDer $\mathrm{f} 1$ vaccine delivered by the MHC class II pathway of antigen presentation and analysis of the effectiveness for specific immunotherapy. Int J Clin Exp Pathol. 2014,7(8): 4636-4644.

[3] Akdis M, Akdis CA. Mechanisms of allergen-specific immunotherapy. J Allergy Clin Immunol. 2007,119(4): 780-791.

[4] Bohm L, Maxeiner J, Meyer-Martin H, et al. IL-10 and Regulatory T cells Cooperate in Allergen-Specific Immunotherapy To ameliorate Allergic Asthma. J Immunol. .2015.194(3): 887-897.

[5] Cui Y. Structural biology of mite allergens [J] . Mol Biol Rep. 2013, 40(1): 681-686.

[6] Hansen G, Berry G, DeKruyff RH, et al. Allergen-specific Th1 cells fail to counterbalance Th2 cell-induced airway hyperreactivity but cause severe airway inflammation [J].J Clin Invest.1999,103 (2):175183.

[7] Maggi E. The TH1/TH2 paradigm in allergy[J]. Immunotechnology. 1998, 3(4):233-244.

[8] Shi YH, Shi GC, Wan HY, et al. Coexistence of Th1/Th2 and Th17/Treg imbalances in patients with allergic asthma [J]. Chin Med J (Engl). 2011, 124(13):1951-1956. 
[9] Zhao J, Lloyd CM., Noble A. Th17 responses in chronic allergic airway inflammation abrogate regulatory T-cell-mediated tolerance and contribute to airway remodeling[J]. Mucosal Immunol. 2013,6(2): 335-346.

[10] Thomas WR, Hales BJ, Smith WA. House dust mite allergens in asthma and allergy[J]. Trends Mol Med. 2010,16(7): 321-328.

[11] Moller C, Dreborg S, Ferdousi HA, et al. Pollen immunotherapy reduces the development asthma inchildren with seasonal rhinoconjunctivitis (the PAT-study). J Allergy Clin Immunol, 2002, 109(2): 251-256.

[12] Minematsu N, Nakamura H, Furuuchi M, et al. Common functional polymorphisms in the cathepsin S promoter in Japanese subjects: possible contribution to pulmonary emphysema [J]. Respirology, 2008, 13(4): 498-504.
[13] Steinman RM. Decisions about dendritic cells: past, present and future [J].Annu Rev Immunol,2012,30(1):1-22.

[14] Caminschi I and Shortman K. Boosting antibody responses by targeting antigens to dendritic cells [J]. Trends Immunol, 2012,33(2):71-77.

[15] Takeda M, Ito W, Tanabe M, Ueki S, Kihara J, Kato H, et al. The pathophysiological roles of PI3Ks and therapeutic potential of selective inhibitors in allergic inflammation. Int Arch Allergy Immunol. 2010,152(Sup 1):90-95.

[16] Qi S, Xin Y, Guo Y, et al. Ampelopsin reduces endotoxic inflammation via repressing ROS-mediated activation of PI3K/Akt/NF-kappaB signaling pathways. Int Immunopharmacol, 2012,12(1):278-87. 